\title{
Some links between extremum spanning forests, watersheds and min-cuts
}

\author{
Cédric Allène ${ }^{\mathrm{a}, \mathrm{b}}$, Jean-Yves Audibert ${ }^{\mathrm{a}}$, Michel Couprie $^{\mathrm{b}}$ and \\ Renaud Keriven ${ }^{\text {a }}$ \\ ${ }^{a}$ Université Paris-Est, CERTIS, Ecole des Ponts ParisTech, France \\ ${ }^{\mathrm{b}}$ Université Paris-Est, Laboratoire d'Informatique Gaspard Monge, Équipe A3SI, \\ ESIEE Paris, France
}

\begin{abstract}
Minimum cuts, extremum spanning forests and watersheds have been used as the basis for powerful image segmentation procedures. In this paper, we present some results about the links which exist between these different approaches. Especially, we show that extremum spanning forests are particular cases of watersheds from arbitrary markers and that min-cuts coincide with extremum spanning forests for some particular weight functions.
\end{abstract}

Key words: Min-cuts, extremum spanning forests, watersheds, segmentation. PACS: 01.30.-y

\section{Introduction}

Min-cuts (graph cuts) and watersheds are two popular tools for image segmentation, which can both be expressed in the framework of graphs and are well suited to computer implementations. Informally, a cut in a connected graph is a set of edges which, when removed from the graph, separates it into several connected components. Given a set of nodes or subgraphs called markers, the

Email addresses: allene@certis.enpc.fr (Cédric Allène), audibert@certis.enpc.fr (Jean-Yves Audibert), m.couprie@esiee.fr (Michel Couprie), keriven@certis.enpc.fr (Renaud Keriven).

URLs: http://certis.enpc.fr/〜allene (Cédric Allène), http://certis.enpc.fr/ audibert (Jean-Yves Audibert), http://www.esiee.fr/ ${ }^{\sim}$ coupriem (Michel Couprie), http://certis.enpc.fr/ keriven (Renaud Keriven). 
goal of these operators is to find a cut for which each induced component contains exactly one marker and which best matches a criterion based on the image contents. For example, the criterion is often designed in such a way that the cut is located along the contours of the objects present in the image. To this end, edges of the pixel adjacency graph can be weighted for example with the inverse of the gradient modulus. The principle of min-cut segmentation is then to find a cut (relative to the markers) whose sum of edge weights is minimal [1].

The watershed is a well-known notion from the field of topography, introduced for image segmentation purposes by S. Beucher and C. Lantuéjoul [2]. Intuitively, the watershed of a function (seen as a topographical surface) is composed by the locations from which a drop of water could flow down towards different minima. In a framework of edge-weighted graphs, the watershed is defined in [3,4] as a cut relative to the regional minima of the weight function, and which satisfies this "drop of water" principle. In [5], F. Meyer shows the link between minimum spanning forests and flooding algorithms, which are most often used to compute watersheds. There is indeed an equivalence between watersheds defined as cuts satisfying the drop of water principle and cuts induced by minimum spanning forests (MinSF) relative to the minima, as proved in $[3,4]$.

The goal of this paper is to clarify the links between these different optimal structures used for image segmentation. For this purpose, we first give a set of definitions for these different paradigms in a same unifying framework of edge-weighted graphs. We show that the cuts induced by MinSFs are particular cases of watersheds from arbitrary markers. Furthermore, these particular cases are often preferable in practice to the watersheds. At last, we prove a property which links graph cuts and watersheds, through the notion of MinSF. It is well known that the MinSFs, and hence the watersheds, are invariant if an increasing transformation is applied simultaneously to all the weights. For example, if we raise all the weights to a same positive power $n$, a MinSF remains a MinSF. On the contrary, min-cuts may be different for different values of $n$. We show that, for any weighted graph, there exists a value $n$ such that min-cuts coincide with cuts induced by maximum spanning forests relative to the markers, furthermore, this will also be true for any number greater than $n$.

The results of section 6 of this paper were stated, without proof, in a conference paper [6]. In this conference paper, a link between MinSFs and a framework based on shortest path forests was also established. This aspect is developed in [4]. Proofs of the theorems (respectively, 5.2, 5.3 and 6.1) are given in the appendices (respectively, A, B and C). 


\section{Basic notions on graphs}

In this section, we state basic notions on graphs before presenting the definitions of extension and cut over a graph, which will be necessary in the sequel of the paper.

We define a graph as a pair $G=(V, E)$ where $V$ is a finite set and $E$ is composed of unordered pairs of elements of $V$, precisely, $E$ is a subset of $\{\{x, y\} \subseteq V \mid x \neq y\}$. Each element of $V$ is called a node or a vertex (of $G$ ) and each element of $E$ is called an edge (of $G$ ). We denote by $G_{\emptyset}$ the empty graph, i.e. $G_{\emptyset}=(\emptyset, \emptyset)$.

Let $X=\left(V^{\prime}, E^{\prime}\right)$ be a graph. If $V^{\prime} \subseteq V$ and $E^{\prime} \subseteq E$ then we say that $X$ is a subgraph of $G$ and we write $X \subseteq G$. Notice that $G_{\emptyset}$ is a subgraph of any graph.

Let $X$ be a subgraph of $G$. We denote, respectively, by $V(X)$ and $E(X)$ the node set and the edge set of $X$.

If $e=\{x, y\}$ is an edge of $G$, we say that $x$ and $y$ are adjacent (in $G$ ). If moreover $x \in V(X)$, where $X$ is a subgraph of $G$, and $y \notin V(X)$, we say that $y$ is adjacent to $X$ (in $G$ ).

Let $\pi=\left\langle x_{0}, \ldots, x_{\ell}\right\rangle$ be an ordered sequence of nodes of $G$, we say that $\pi$ is a path from $x_{0}$ to $x_{\ell}$ in $G$ (or in $V$ ) if for any $i \in[1 ; \ell], x_{i}$ is adjacent to $x_{i-1}$. In this case, we say that $x_{0}$ and $x_{\ell}$ are linked for $G$.

We say that $\pi$ is a simple path from $x_{0}$ to $x_{\ell}$ in $G$ (or in $V$ ) if $\pi$ is a path from $x_{0}$ to $x_{\ell}$ and if all nodes of $\pi$ are distinct. Notice that if there exists a path from $x_{0}$ to $x_{\ell}$ in $G$, then there exists a simple path from $x_{0}$ to $x_{\ell}$. We say that $G$ is connected if any two vertices of $G$ are linked for $G$. Notice that $G_{\emptyset}$ is connected.

Notice that you can define a path through its edges: $\pi=\left\langle e_{0}, \ldots, e_{\ell-1}\right\rangle$ with $e_{0}=\left\{x_{0}, x_{1}\right\}, \ldots, e_{\ell-1}=\left\{x_{\ell-1}, x_{\ell}\right\}$. Then, we say that $\pi$ is a path either from $x_{0}$ to $x_{\ell}$ or from $e_{0}$ to $e_{\ell-1}$ in $G$ (or in $E$ ).

We say that $X$ is a connected component of $G$ if $X$ is a connected subgraph of $G$ which is maximal for this property, i.e. for any connected graph $X^{\prime}$, $X \subseteq X^{\prime} \subseteq G$ implies $X^{\prime}=X$. Notice that $G_{\emptyset}$ is not a connected component of any non-empty graph, and that $G_{\emptyset}$ is the connected component of, and only of, $G_{\emptyset}$.

Let $X$ and $Y$ be two subgraphs of $G$. We define $(X \cup Y)=(V(X) \cup V(Y), E(X) \cup$ $E(Y)$ and $(X \cap Y)=(V(X) \cap V(Y), E(X) \cap E(Y))$. 
Let $X$ be a subgraph of $G$. An edge $e=\{x, y\}$ of $G$ is adjacent to $X$ if $\{x, y\} \cap V(X) \neq \emptyset$ and $e \notin E(X)$. Consequently, if $x \in V(X)$ :

- either $y \in V(X)$,

- or $y$ is adjacent to $X$, in this case, we say that $e$ is strictly adjacent to $X$.

If $S$ is a subset of $E$, we denote by $\bar{S}$ the complementary set of $S$ in $E$, that is, $\bar{S}=E \backslash S$.

Let $S \subseteq E$. The graph induced by $S$ (in $G$ ) is the graph whose edge set is $S$ and whose vertex set is made of all points which belong to an edge in $S$.

We now present the notions of extension and (graph) cut which play an important role for optimal structures in edge-weighted graphs. The notion of extension was introduced in [7] for the case of connected graphs. The following definition presents this notion in the case of arbitrary graphs.

\section{Definition 1.1 (Extension, spanning extension, maximal extension and cut)}

Let $G$ be a graph and let $G_{1}, G_{2}, \ldots, G_{n}$ be the connected components of $G$. Let $M$ and $X$ be two subgraphs of $G$. For any $i \in[1 ; n]$, let $M_{i}=M \cap G_{i}$ and $X_{i}=X \cap G_{i}$.

- We say that $X$ is an extension of $M$ if, for all $i \in[1 ; n], M_{i} \subseteq X_{i}$ and each connected component of $X_{i}$ contains exactly one connected component of $M_{i}$.

- We say that $X$ is a spanning extension of $M$ (over $G$ ) if $X$ is an extension of $M$ and if $V(X)=V$.

- We say that $X$ is a maximal extension of $M$ (over $G$ ) if $X$ is an extension of $M$ and if there is no extension of $M$ which strictly contains $X$.

- Let $C \subseteq E$, we say that $C$ is a (graph) cut relative to $M$ (over $G$ ) if $\bar{C}$ is an extension of $M$ over $G$ and if $C$ is minimal for this property (i.e. considering $D \subseteq E$, we have $C=D$ whenever $D \subseteq C$ and $\bar{D}$ is an extension of $M$ over $G)$.

It may be seen that, if $C$ is a cut, then the graph induced by $\bar{C}$ is necessarily a maximal extension. Moreover, if $X$ is a spanning extension of $M$, then there exists a unique cut $C$ relative to $X$ which is called the cut induced by $X$. It may be seen that $C$ is also a cut relative to $M$.

Examples of these definitions are shown in figure 1.

\section{Remark 1.1}

Let $M$ be a subgraph of $G$.

(1) A maximal extension of $M$ is always a spanning extension of $M$ (the converse is not necessarily true since one can have a spanning extension 
to which one can add an edge while keeping the extension property).

(2) For any extension $X$ of $M$, there exists a maximal extension $Y$ of $M$ such that $X \subseteq Y$.

(3) For any $i \in[1, n]$, if $M_{i}=G_{\emptyset}$, then any connected subgraph of $G_{i}$ is an extension of $M_{i}$ and $G_{i}$ is the maximal extension of $M_{i}$.

(4) Each edge of a cut $C$ is adjacent to exactly two connected components of the graph induced by $\bar{C}$.

(5) Notice that, if any connected component of $G$ contains no more than one connected component of $M$, then the unique cut $C$ relative to $M$ is the empty set.

\section{Definition 1.2 (Forest)}

Let $F$ and $M$ be two subgraphs of $G$.

We say that $F$ is a forest relative to $M$ if:

- $F$ is an extension of $M$, and

- for any extension $X \subseteq F$ of $M, V(X)=V(F) \Rightarrow X=F$ (i.e. we cannot eliminate an edge of $F$ and keep the extension property).

\section{Definition 1.3 (Spanning forest)}

Let $F$ and $M$ be two subgraphs of $G$.

We say that $F$ is a spanning forest relative to $M$ (over $G$ ) if:

- $F$ is a forest relative to $M$, and

- $V(F)=V$.

Equivalently, we say that $F$ is a spanning forest relative to $M$ (over $G$ ) if there exists a spanning extension $X$ relative to $M$ over $G$ such that $F$ is obtained by eliminating edges of $X$ as long as it is possible to do it while preserving the spanning extension property. In other words, a spanning forest is a minimal spanning extension.

Examples of these definitions are shown in figures $1 \mathrm{f}$ and $1 \mathrm{~g}$.

It can be seen that if $G$ is connected and $M=\left(V_{M}, \emptyset\right)$ where $V_{M} \subseteq V$ (i.e. $M$ is a subgraph without edge), then the notion of forest relative to $M$ corresponds exactly to the usual notion of forest. Furthermore, if $|V(M)|=1$ then we retrieve the usual notion of tree.

\section{Notions on edge weighted graphs}

In this section, we recall in particular the definition of a Minimum Spanning Forest (MinSF) relative to a subgraph of $G$.

It is shown in [4] that the problem of computing a MinSF is equivalent to the 


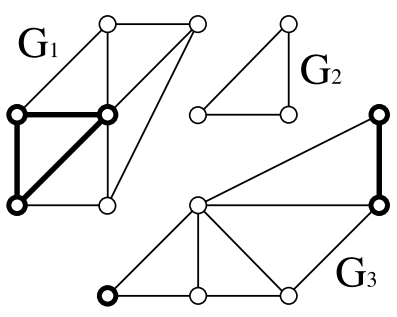

(a)

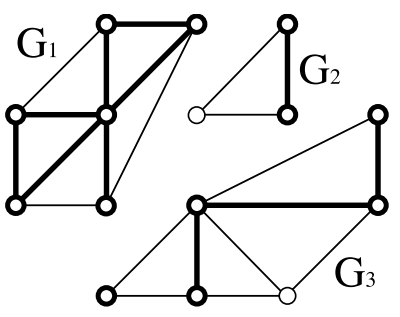

(b)

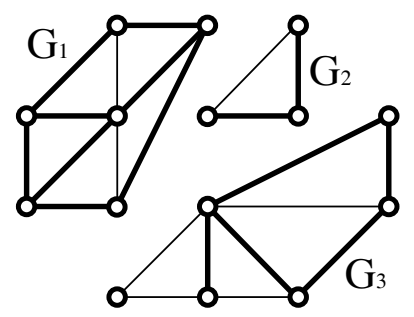

(c)

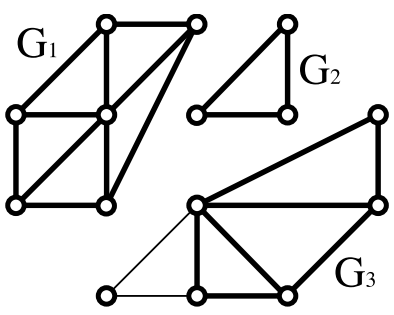

(d)

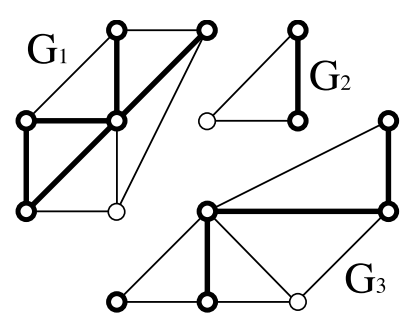

(f)

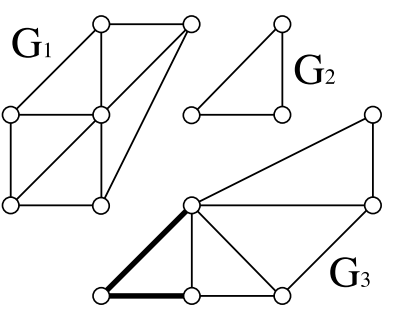

(e)

Figure 1. Graph $G$, composed of three connected components $\left(G_{1}, G_{2}\right.$ and $\left.G_{3}\right)$, with in bold: (a) a subgraph $M$; (b) an extension relative to $M$; (c) a spanning extension relative to $M$; (d) a maximal extension relative to $M$; (e) a cut relative to $M$; (f) a forest relative to $M$; (g) a spanning forest relative to $M$.

one of computing a minimum spanning tree, which has been studied for many years in combinatorial optimization (see $[8,9]$ ).

In the following, $P$ will denote a map from $E$ to $\mathbb{R}^{+}$.

The pair $(G, P)$ is an edge weighted graph. If $e$ is an edge of $G, P(e)$ is called the weight, or the altitude, of $e$. The weight of a subgraph $X$ of $G$, denoted by $P(X)$, is the sum of its edge weights $\left(P(X)=\sum_{x \in E(X)} P(x)\right)$.

\section{Definition 2.1 (Minimum spanning forest)}

Let $F$ and $M$ be two subgraphs of $G$. We say that $F$ is a Minimum Spanning Forest (MinSF) relative to $M$ (for $P$ ) if $F$ is a spanning forest relative to $M$ and if the weight of $F$ is minimum, i.e. lower than or equal to the weight of any other spanning forest relative to $M$.

Notice that if the weight of $F$ is maximum instead of minimum, then we have a Maximum Spanning Forest (MaxSF). 
An example of this definition is shown in figure 9.

\section{Remark 2.1}

Let $M$ and $F$ be two subgraphs of $G, f: \mathbb{R}^{+} \rightarrow \mathbb{R}^{+}$be a strictly increasing function and $g: \mathbb{R}^{+} \rightarrow \mathbb{R}^{+}$be a strictly decreasing function.

From classical results on extremum spanning forests (see [9], chapter 6.1), we know that the three following statements are equivalent:

- $F$ is a MinSF relative to $M$ for $P$;

- $F$ is a MinSF relative to $M$ for $(f \circ P)$;

- $F$ is a MaxSF relative to $M$ for $(g \circ P)$.

Let $M$ be a subgraph of $G$ and let $F$ be a MinSF relative to $M$. Since $F$ is a spanning forest, hence a spanning extension, there exists a unique (graph) cut relative to $M$ induced by $F$. We say that this cut is a MinSF cut relative to $M$.

\section{Definition 2.2 (Threshold of a map)}

For any $h \in \mathbb{R}^{+}$, we define $E_{P}^{h}=\{e \in E ; P(e) \leq h\}$.

Let $X$ be a subgraph of $G$. Then, we denote by $X_{P}^{h}$ the graph induced by $E(X) \cap$ $E_{P}^{h}$ and we say that $X_{P}^{h}$ is the (inferior) threshold of $X$ at level $h$ for $P$. When there is no ambiguity about the map, we will simply write $X^{h}$.

Examples of this definition are shown in figure 2.

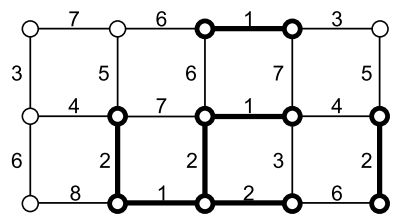

(a)

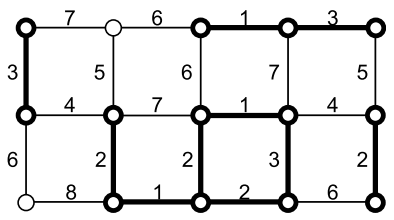

(b)

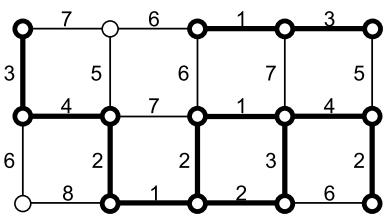

(c)

Figure 2. Graph $G$ and map $P$ with in bold: (a) $G_{P}^{2}$; (b) $G_{P}^{3}$; (c) $G_{P}^{4}$.

\section{Watersheds on edge weighted graphs}

The intuitive idea underlying the notion of watershed comes from the field of topography: a drop of water falling down on a topographic surface follows a descending path and reaches a regional minimum area. The watershed may be thought of as the separating lines of the domain of attraction of drops of water (see $[2,10-12]$ ).

The regions of a watershed, also called catchment basins, are associated with the regional minima of the map. In other words, each catchment basin contains 
a unique regional minimum, and conversely, each regional minimum is included in a unique catchment basin: the regions of the watershed are the connected components of an extension relative to the minima. They are separated by a set of edges from which a drop of water can flow down towards different minima, in the sense defined below.

A subgraph $X$ of $G$ is a (regional) minimum of $P$ if:

- $X$ is connected, and

- all the edges of $X$ have the same altitude, that we will refer to as the altitude of $X$, and

- the altitude of any edge adjacent to $X$ is strictly greater than the altitude of $X$.

We denote by $\operatorname{Min}(P)$ the graph whose vertex set and edge set are, respectively, the union of the vertex sets and edge sets of all minima of $P$.

Let $\pi=\left\langle x_{0}, \ldots, x_{\ell}\right\rangle$ be a path in $G$. The path $\pi$ is descending (for $P$ ) if $\forall i \in\{1, \ldots, \ell-1\}, P\left(\left\{x_{i-1}, x_{i}\right\}\right) \geq P\left(\left\{x_{i}, x_{i+1}\right\}\right)$.

\section{Definition 3.1 (Watershed - Def. 3 in [3])}

Let $C$ be a subset of $E$. We say that $C$ is a watershed cut (for $P$ ), or simply a watershed (for $P$ ), if the graph induced by $\bar{C}$ is a maximal extension of $\operatorname{Min}(P)$ and if for any $e=\left\{x_{0}, y_{0}\right\} \in C$, there exist $\pi_{1}=\left\langle x_{0}, \ldots, x_{m}\right\rangle$ and $\pi_{2}=\left\langle y_{0}, \ldots, y_{n}\right\rangle$ two descending paths in $\bar{C}$ such that:

- $x_{m}$ and $y_{n}$ are nodes of two distinct minima of $P$, and

- $P(e) \geq P\left(\left\{x_{0}, x_{1}\right\}\right)$ (respectively, $P(e) \geq P\left(\left\{y_{0}, y_{1}\right\}\right)$ ), whenever $m>0$ (respectively, $n>0)$.

The connected components of $\bar{C}$ are the catchment basins of this watershed.

An example of this definition is shown in figure 9 .

Notice that a watershed is indeed a graph cut relative to $\operatorname{Min}(P)$.

\section{Watersheds relative to markers}

Usually, when one wants to compute a watershed of a map, one obtains an over-segmented result since the number of regions in the final result is equal to the number of regional minima. Sometimes, it is desirable to use a set of markers instead of the minima of the map to fix the number of regions and mark some vertices with a specific label. Traditionally, to do this, one has to proceed to a morphological reconstruction of the map before computing the watershed. This reconstruction is needed on the map $P$ for a subgraph $M$ of $G$ to become the minima of a new map $P^{\prime}$. As a consequence, we have 
$M=\operatorname{Min}\left(P^{\prime}\right)$ and, then, it is possible to compute a watershed algorithm from the marker $M$ (see $[10,11])$.

\section{Definition 4.1 (Flooding maps [13])}

We say that the map $P^{\prime}$ is a flooding map of $P$ (for $G$ ) if $\forall h \in \mathbb{R}^{+}$, any connected component of $G_{P^{\prime}}^{h}$ is a connected component of $G_{P}^{h}$.

We denote by $\mathcal{F}(P)$ the set of flooding maps of $P$.

Examples of this definition are shown in figures 3 and 4 .

Notice that, by definition of a flooding map $P^{\prime}$ of $P$ (for $G$ ), we have $\forall e \in$ $E, P^{\prime}(e) \geq P(e)$.

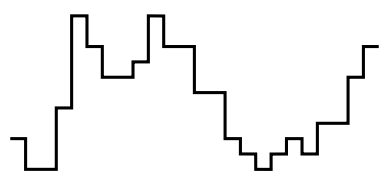

(a)

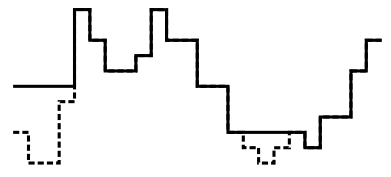

(b)

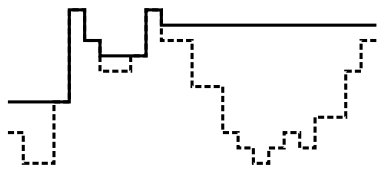

(c)

Figure 3. Representation in 1D of: (a) a map $P$; (b,c) flooding maps of $P$ (with $P$ in dashed line).

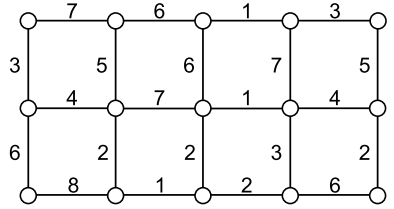

(a)

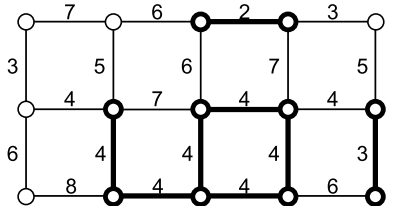

(b)

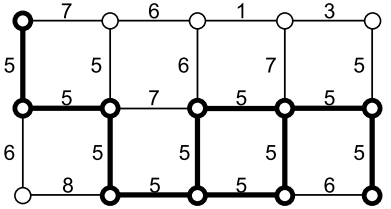

(c)

Figure 4. Graph $G$ with: (a) a map $P$; (b,c) flooding maps of $P$ (with edges of different weight in bold).

\section{Definition 4.2 (Constrained flooding maps)}

Let $M$ be a subgraph of $G$.

We say that the map $P^{\prime}$ is a flooding map of $P$ constrained by $M$ if:

- $P^{\prime}$ is a flooding map of $P\left(P^{\prime} \in \mathcal{F}(P)\right)$, and

- $\forall e \in E(M), P(e)=P^{\prime}(e)$.

We denote by $\mathcal{F}(P, M)$ the set of flooding maps of $P$ constrained by $M$.

Examples of this definition are shown in figures 5 and 6 .

\section{Definition 4.3 (Relative watershed)}

Let $M$ be a subgraph of $G$ and let $C$ be a cut relative to $M$.

We define the map $P^{\prime}$ such that:

$$
\forall e \in E, P^{\prime}(e)= \begin{cases}\alpha & \text { if } e \in E(M) \\ P(e) & \text { otherwise }\end{cases}
$$




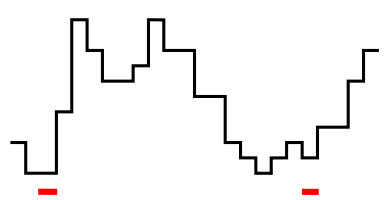

(a)

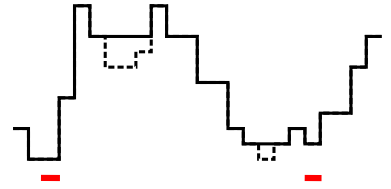

(b)

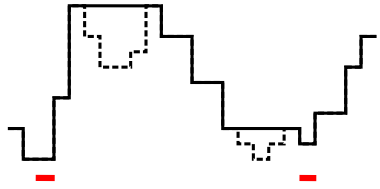

(c)

Figure 5. Representation in 1D of: (a) a map $P$ with markers $M$ (in red); (b) a flooding map of $P$ constrained by $M$ (with $P$ in dashed line); (c) a maximum flooding map of $P$ constrained by $M$ (with $P$ in dashed line).

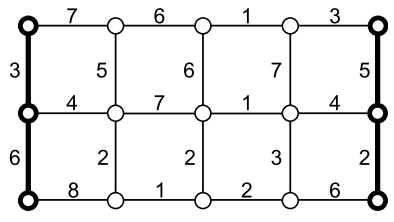

(a)

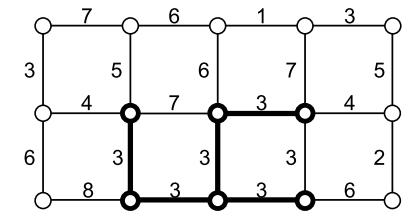

(b)

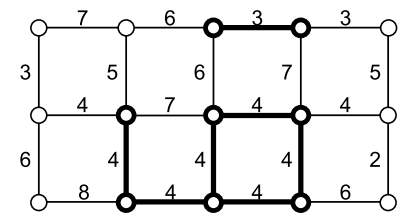

(c)

Figure 6. Graph $G$ with: (a) a map $P$ and, in bold, a subgraph $M$ in bold; (b,c) flooding maps of $P$ constrained by $M$ (with edges of different weight in bold).

with $\alpha \leq \min _{e \in E}(P(e))$, and the map $P^{\prime \prime}$ as the maximum flooding map of $P^{\prime}$ constrained by $M$ :

$$
P^{\prime \prime}=\max _{p \in \mathcal{F}\left(P^{\prime}, M\right)}(p) \text {. }
$$

We say that $C$ is a watershed relative to $M$ (for $P$ ) if $C$ is a watershed for $P^{\prime \prime}$.

Examples of this definition are shown in figures 7 and 8 .

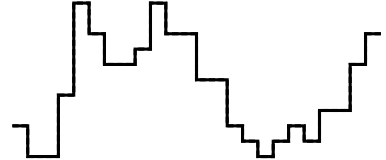

(a)

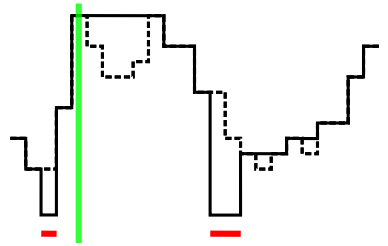

(d)

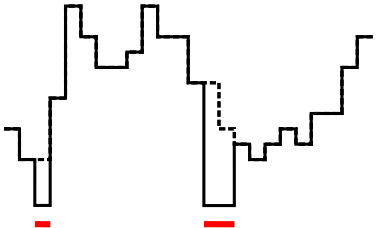

(b)

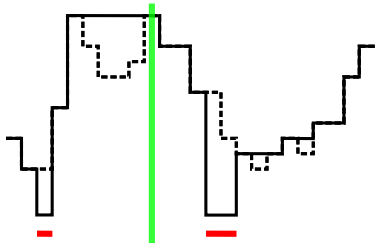

(e)

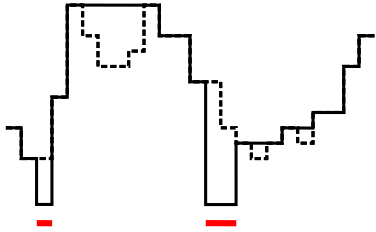

(c)

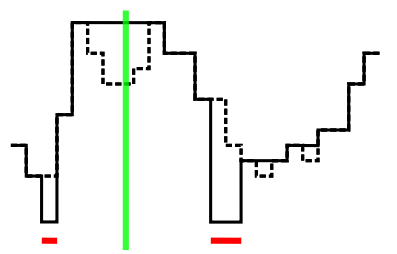

(f)

Figure 7. Representation in 1D of: (a) map $P$ with markers $M$ (in red); (b) map $P^{\prime}$ with markers $M$ as lowest weights (with $P$ in dashed line); (c) maximum flooding map of $P^{\prime}$ constrained by $M$ (with $P$ in dashed line); (d) to (f) three different watersheds (in green) relative to $M$. 


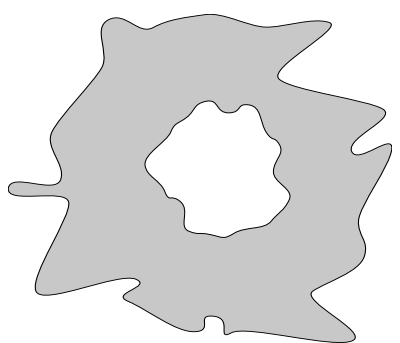

(a)

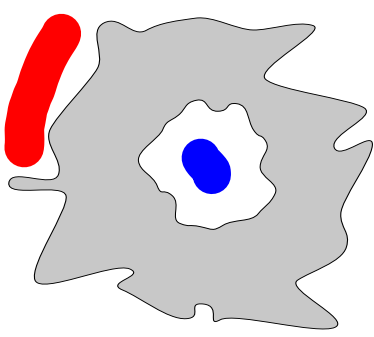

(b)

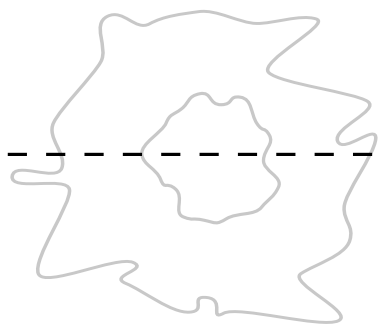

(c)

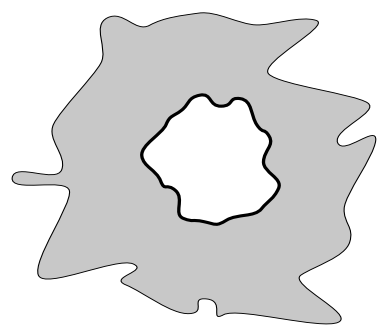

(f)

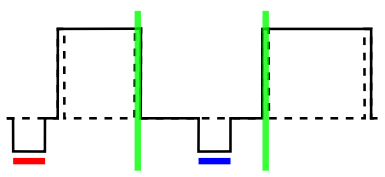

(i)

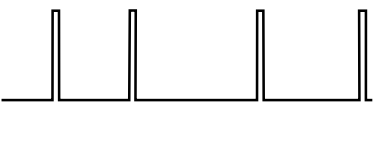

(d)

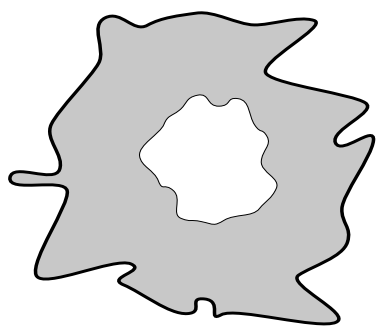

$(\mathrm{g})$

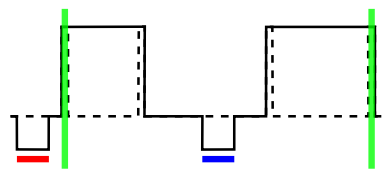

(j)

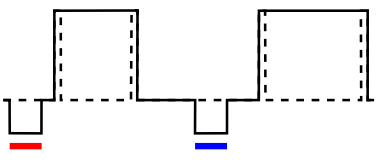

(e)

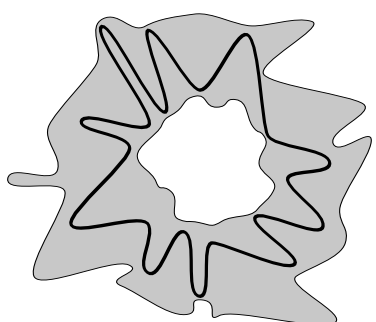

(h)

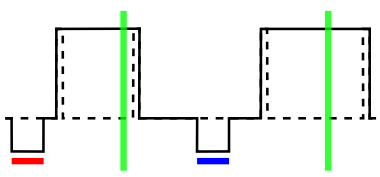

$(\mathrm{k})$

Figure 8. Relative watershed on a gradient based weight function: (a) input image; (b) markers (two sets: red and blue); (c) gradient value of the image; (d) 1D profile of the gradient (represented by the dashed line on (c)); (e) 1D profile of the maximum flooding map of the gradient with markers as minimum values; (f) to (h) watershed results; (i) to (k) 1D profiles of watershed results (respectively (f) to (h)).

This morphological reconstruction is often done on node weighted graphs. Its best algorithm is quasi-linear (see [14]).

Unfortunately, this method gives in some cases unexpected results since the watershed relative to the markers could pass through a catchment basin of the watershed (relative to the minima) (see figures $7 \mathrm{f}, 8 \mathrm{k}$ and $8 \mathrm{~h}$ ), or worse, pass through a minimum of the original map. 


\section{Relations between MinSF cuts and watersheds}

In this section, we recall the equivalence between MinSF cuts and watersheds relative to the minima of a map (see [3]) before describing a new theorem stating that a MinSF cut relative to any marker is a relative watershed.

Theorem 5.1 (Th. 2 in [3])

Let $C$ be a subset of $E$.

The set $C$ is a MinSF cut relative to $\operatorname{Min}(P)$ (for $P$ ) if and only if $C$ is a watershed (for $P$ ).

An illustration of this theorem is shown in figure 9 .

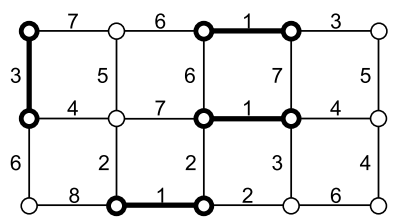

(a)

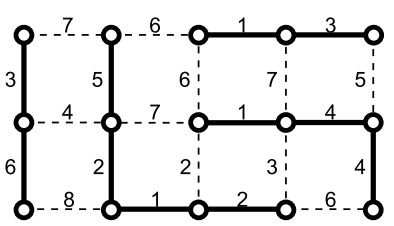

(b)

Figure 9. Graph $G$ and map $P$ with: (a) a subgraph $M=\operatorname{Min}(P)$ in bold; (b) a MinSF relative to $M$ for $P$ in bold and its induced cut $C$ in dashed edges. It can be seen, from definition 3.1, that $C$ is indeed a watershed.

The two following theorems lead us to the conclusion that reconstruction plus watershed can be advantageously replaced by a single MinSF computation for doing a segmentation from markers.

\section{Theorem 5.2}

Let $M$ be a subgraph of $G$ and $P^{\prime}$ a flooding map of $P\left(P^{\prime} \in \mathcal{F}(P)\right)$.

If $F$ is a MinSF relative to $M$ over $G$ for $P$, then $F$ is a MinSF relative to $M$ over $G$ for $P^{\prime}$.

An illustration of this theorem is shown in figure 10 .

\section{Remark 5.1}

Notice that the converse of theorem 5.2 is, in general, not true (see figures $10 j$ and $10 \mathrm{k})$.

\section{Theorem 5.3}

Let $M$ be a subgraph of $G$ and $C$ be a subset of $E$.

If the set $C$ is a MinSF cut relative to $M$ (for $P$ ), then $C$ is a watershed relative to $M$ (for $P$ ).

An illustration of this theorem is shown in figure 11. 


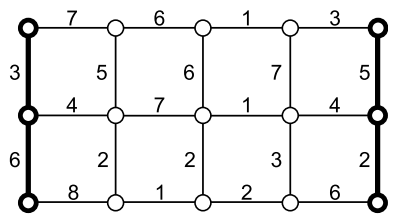

(a)

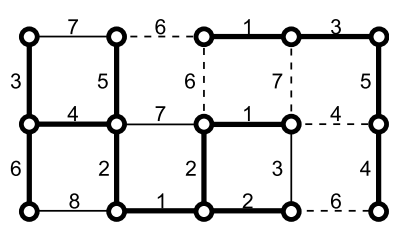

(d)

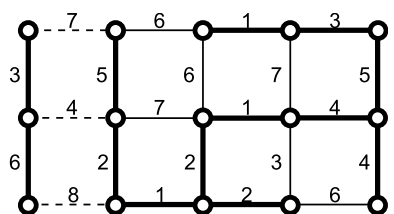

(g)

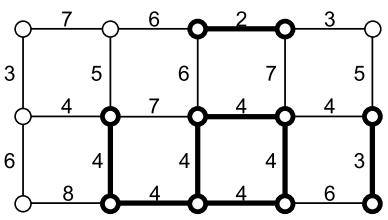

(b)

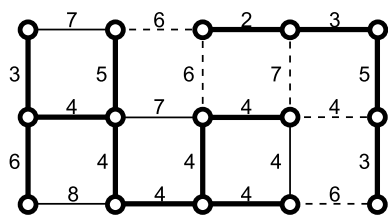

(e)

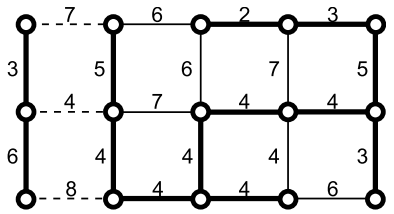

(h)

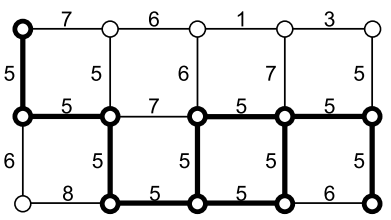

(c)

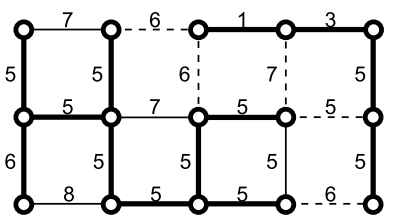

(f)

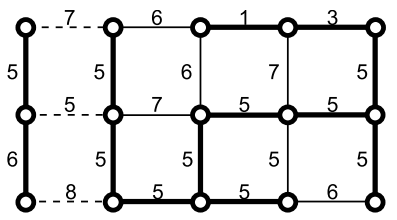

(i)

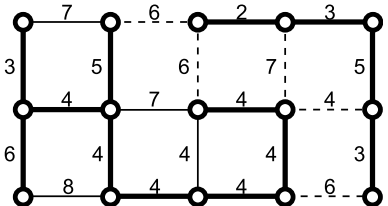

(j)

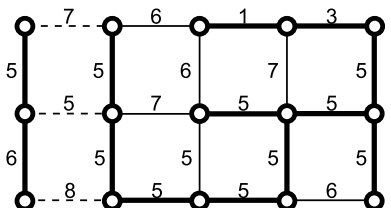

(k)

Figure 10. Graph $G$ with: (a) a map $P$ and, in bold, a subgraph $M$; (b,c) flooding maps, respectively $P^{\prime}$ and $P^{\prime \prime}$, of $P$ (with edges of different weight in bold); (d) to (f) MinSF $F_{1}$ relative to $M$ over $G$ for, respectively, $P, P^{\prime}$ and $P^{\prime \prime}$; (g) to (i) MinSF $F_{2}$ relative to $M$ over $G$ for, respectively, $P, P^{\prime}$ and $P^{\prime \prime}$; (j,k) MinSF relative to $M$ over $G$ for, respectively, $P^{\prime}$ and $P^{\prime \prime}$ but not for $P$. In dashed edges are the cuts induced by the MinSFs.

\section{Remark 5.2}

Notice that the converse of theorem 5.3 is, in general, not true (see figures $11 \mathrm{~h}$ to 11l).

Any minimum spanning tree algorithm can be employed to compute a MinSF and, as a consequence, a watershed (see a survey in $[8,9]$ ). The best of them does this in quasi-linear time (see [15]). Algorithms specific to watersheds run in linear time (see [3]).

As mentioned above, a relative watershed could lead the cut to pass through a catchment basin or even a minimum of the original map (see figures $11 \mathrm{~h}$ to $11 \ell$ ), whereas a MinSF cut could not. In figure 8, only results of figures $8 \mathrm{f}$ and $8 \mathrm{~g}$ can be obtained with a MinSF computation. 


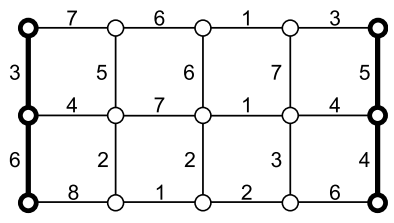

(a)

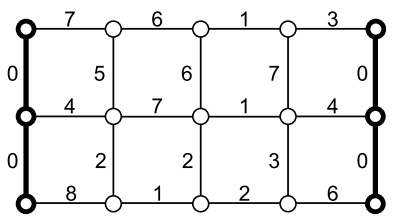

(b)

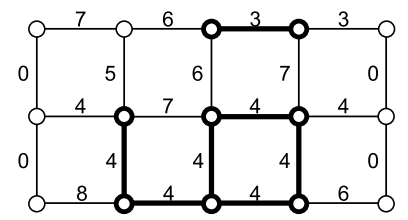

(c)

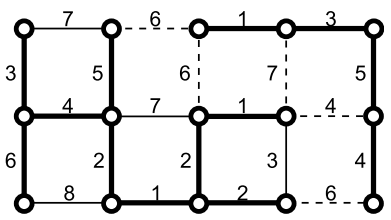

(d)

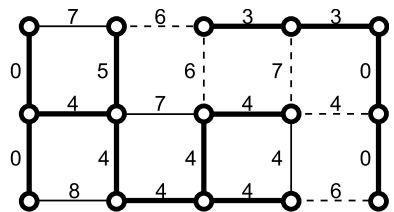

(f)

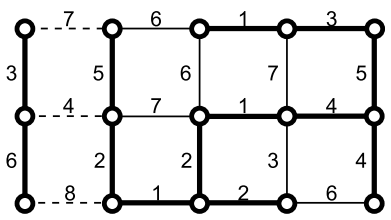

(e)

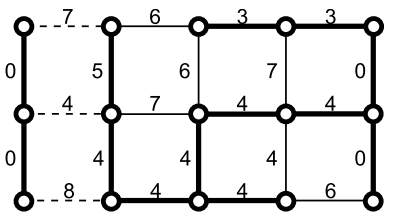

(g)

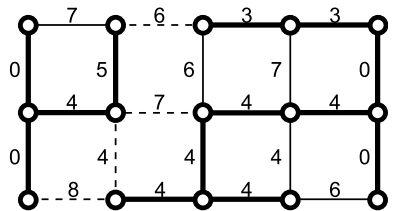

(h)

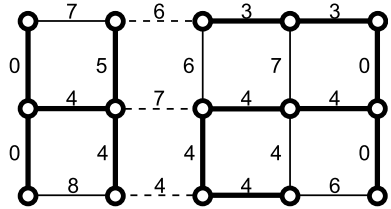

(i)

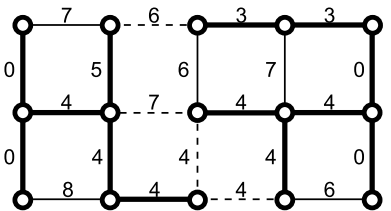

(j)

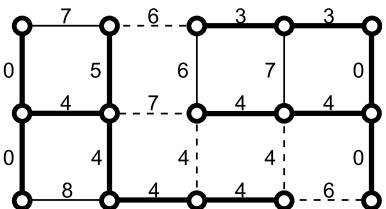

(k)

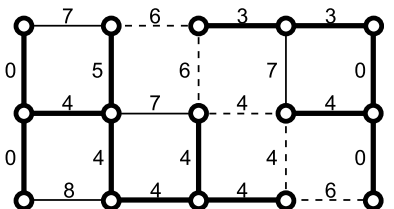

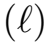

Figure 11. Graph $G$ with: (a) a map $P$ and, in bold, a subgraph $M$; (b,c) respectively maps $P^{\prime}$ and $P^{\prime \prime}$ as described in definition 4.3 (with edges of different weights in bold); (d,e) respectively $F_{1}$ and $F_{2}$ which are both MinSFs relative to $M$ over $G$ for $P$; $(\mathrm{f}, \mathrm{g})$ respectively $F_{1}$ and $F_{2}$ which are both MinSFs relative to $M$ over $G$ for $P^{\prime \prime}$ as well; (h) to (l) MinSFs relative to $M$ over $G$ for $P^{\prime \prime}$ but not for $P$. In dashed edges are the cuts induced by MinSFs which are also watersheds relative to $M$.

In cases where pertinent cuts should follow the crests of the original map, a MinSF should be used rather than a relative watershed. This method is also of interest for implementation of hierarchical segmentation schemes (see [5,12, 16]). Otherwise, there exists strategies to get watersheds which are "centered" in plateaus that are created by the morphological reconstruction (see [10]). 


\section{Link between minimum cuts (min-cuts) and MaxSF cuts}

In this section, we show that min-cuts and MaxSF cuts are linked through a modification of the map $P$ preserving the order and increasing the weight difference between the edges. Let us first recall the notion of minimum cut.

Let $M$ be a subgraph of $G$ and let $C \subseteq E$. We say that $C$ is a minimum cut (min-cut) relative to $M$ (for $P$ ) if for any cut $C^{\prime} \subseteq E$ relative to $M$, $P(C) \leq P\left(C^{\prime}\right)$. It can be seen that a cut $C$ relative to $M$ is of minimum weight if and only if $\bar{C}$ is a (spanning) extension of maximum weight relative to $M$. Examples of this definition are shown in figure 13.

A fundamental result in combinatorial optimization states that, given two isolated nodes of an edge-weighted graph (called source and sink), finding a min-cut that separates these two nodes is equivalent to finding a maximum flow between them (see [17], chapter 6.2). This problem is equivalent to finding a min-cut relative to a subgraph having exactly two connected components (consider adding two extra nodes to $G$, the source and the sink, and highly weighted edges from each one of them to all the nodes of each of the components of $M$ ). The first algorithm to solve the maximum flow problem was created by L. R. S. Ford and D. R. Fulkerson [18] in 1956. Specific variants of maximum flow algorithms have been developed (e.g. for computer vision applications) which allow fast computation [19]. On the other hand, finding a min-cut relative to a subgraph with more than two connected components is NP-hard [20], but there exists approximation algorithms [1].

We denote by $P^{[\alpha]}$, and say $P$ to the power $\alpha$, the map from $E$ to $\mathbb{R}^{+}$defined by, for any $e \in E, P^{[\alpha]}(e)=[P(e)]^{\alpha}$.

\section{Theorem 6.1}

If $M$ is a subgraph of $G$, then there exists a real number $\beta$ such that, for any $\alpha \geq \beta$, any min-cut relative to $M$ for $P^{[\alpha]}$ is a MaxSF cut relative to $M$ for $P^{[\alpha]}$.

Theorem 6.1 is illustrated in figures 12 and 13.

It has to be noticed that the converse of theorem 6.1 is, in general, not true. See figure 15 where the MaxSF cut relative to $M$ for $P$ in $15 \mathrm{~b}$ is not a min-cut relative to $M$ for $P$, but any min-cut is a MaxSF cut.

From remark 2.1, we know that the MaxSF cut relative to $M$ for $P^{[\alpha]}$ is also a MaxSF cut relative to $M$ for $P$ and conversely since the change of map preserves the order. An intuitive interpretation of theorem 6.1 is to consider 


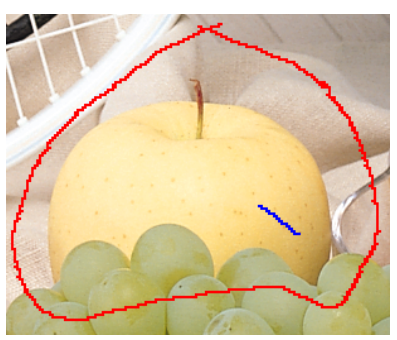

(a)

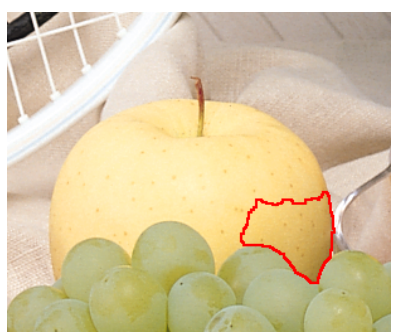

(d)

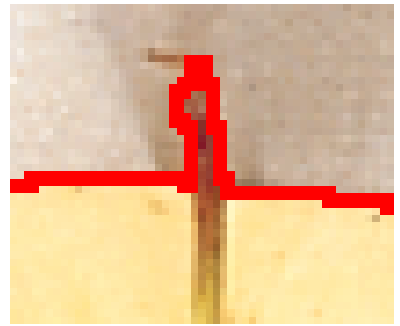

$(\mathrm{g})$

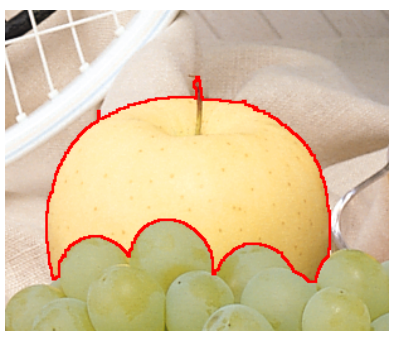

(b)

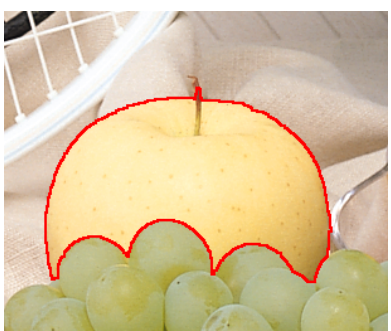

(e)

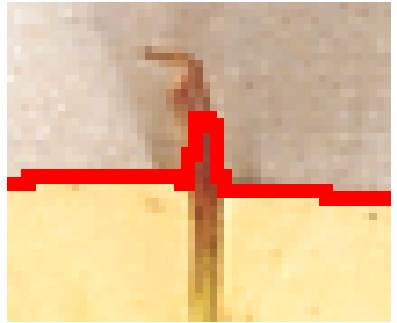

(h)

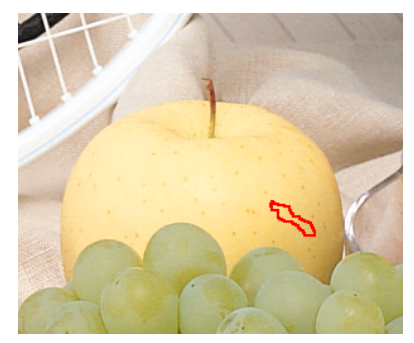

(c)

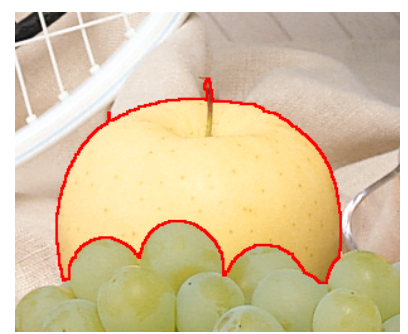

(f)

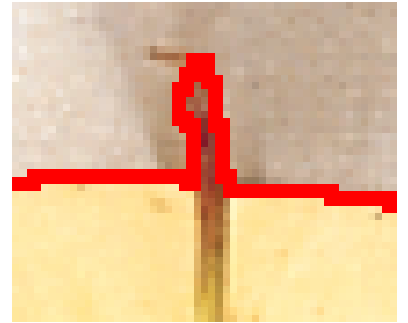

(i)

Figure 12. Color image segmentation using: (a) markers superimposed to the original image; (b) MaxSF cut on $P$; (c) min-cut on $P$; (d) min-cut on $P^{[1.4]}$; (e) min-cut on $P^{[2]}$; (f) min-cut on $P^{[3]}$; (g) zoom of MaxSF cut on $P$; (h) zoom of min-cut on $P^{[2]}$; (i) zoom of min-cut on $P^{[3]}$.

the MaxSF cut as a greedy heuristic to obtain a min-cut. The efficiency of this heuristic becomes higher when differences between the weights increase. More formally, the min cut method (with power $\alpha$ as a parameter) can be considered as a general scheme in which MinSF is a particular case. When the power $\alpha$ is high enough, MinSF cuts are a good alternative to min cuts because of the low computational cost for an exact result with an unlimited number of markers. However, in other cases, a min cut computation cannot be replaced by a MinSF cut computation.

Figure 12 illustrates the link between these two well known segmentation paradigms through the evolution of the min-cut with different values of $\alpha$. Notice that the power of the map $P$ could then be considered as a smoothing term for the min-cut method. Indeed, when this power decreases, shortest cuts are found whereas, when it increases, longer cuts are found. These longer cuts 


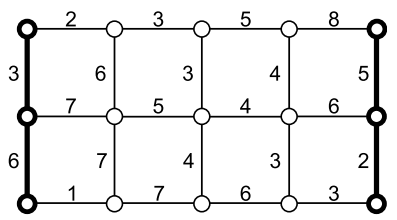

(a)

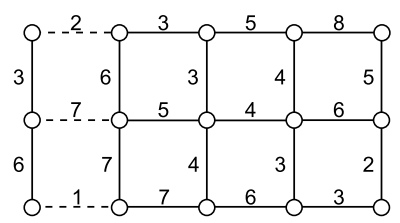

(c)

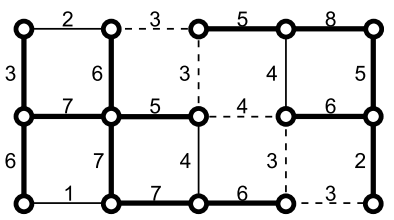

(b)

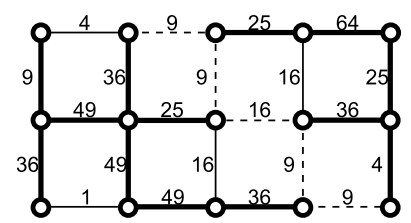

(d)

Figure 13. Graph $G$ and map $P$ with: (a) in bold, a subgraph $M$; (b) in bold, the MaxSF relative to $M$ for $P$ and, in dashed edges, its induced cut; (c) in dashed edges, the min-cut relative to $M$ for $P$; (d) in bold, the MaxSF relative to $M$ for $P^{[2]}$ and, in dashed edges, its induced cut which is also the min-cut relative to $M$ for $P^{[2]}$.

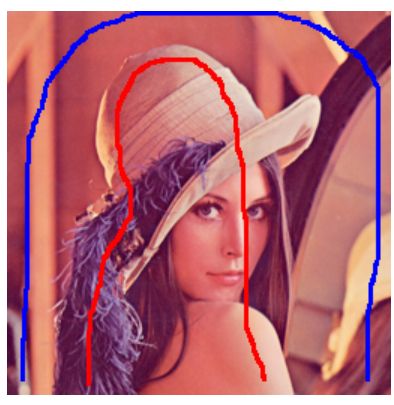

(a)

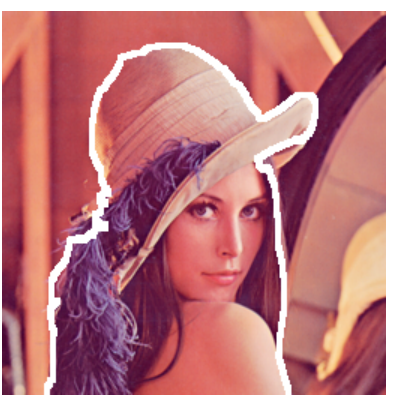

(b)

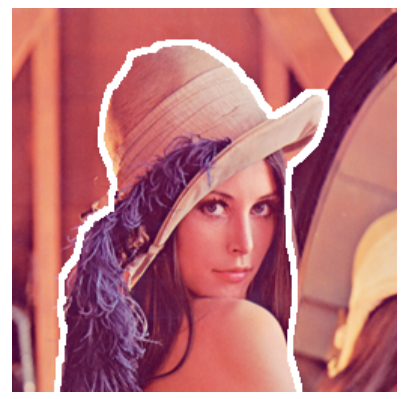

(c)

Figure 14. Color image segmentation: (a) markers superimposed to the original image; (b) MaxSF cut on $P$; (c) min-cut on $P$.

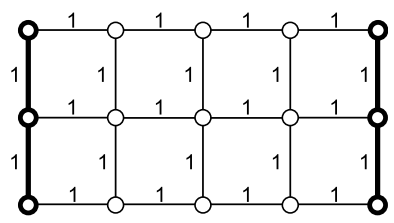

(a)

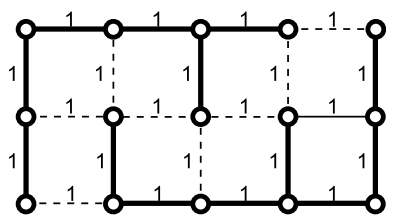

(b)

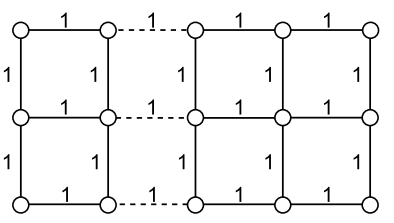

(c)

Figure 15. Graph $G$ and map $P$ with: (a) in bold, a subgraph $M$; (b) in bold, a MaxSF relative to $M$ for $P$ and, in dashed edges, its induced cut, which is not a min-cut; (c) in dashed edges, a min-cut relative to $M$ for $P$.

can surround more details as well as noise. Therefore, releasing this smoothing term is not always suitable. See, for example, figure 14 where the min-cut result can be considered as better than the MaxSF cut result. 


\section{Conclusion}

In the framework of edge-weighted graphs, the notions of minimum spanning forest relative to the minima of a map and watershed are equivalent $[3,4]$. The first contribution of this paper is to clarify the extension of this result to relative watersheds, that is, watersheds from arbitrary markers. We proved that any relative MinSF is a relative watershed, but the converse is not true. Furthermore, the relative watersheds which are not MinSFs are not interesting for usual segmentation tasks, thus MinSFs should in most practical cases be preferred to relative watersheds. The second contribution of this paper is a link between MinSFs (and thus also watersheds) and minimum cuts in edgeweighted graphs. Although different in general, we proved that both notions coincide for particular weight functions.

It can be noticed that the $\beta$ in theorem 6.1 that we provide in the proof (see appendix $\mathrm{C}$ ) as sufficient can be a very large number while, experimentally, quite smaller bounds are usually sufficient in practical cases. Finding a lower bound for $\beta$ remains an open problem. 


\section{A Proof of theorem 5.2}

To prove theorem 5.2, we will use a well-known characterization of minimum spanning trees on a connected graph (proposition A.1) to obtain a characterization of MinSFs (proposition A.2). Then we show that this characterization is preserved through flooding maps (proposition A.3).

Considering that $G$ is connected, let $T$ be a spanning tree over $G$. For any $e=\{x, y\} \in(E \backslash E(T))$, we denote by $\pi_{(T, e)}$ the unique simple path in $T$ going from $x$ to $y$ (i.e. $\pi_{(T, e)}=\langle x, y, \ldots, x\rangle$ ).

Proposition A.1 [Minimum spanning tree property - In [9], chapter 6.1] The spanning tree $T$ over $G$ is a minimum spanning tree over $G$ for $P$ if and only if $\forall e \in(E \backslash E(T)), P(e)=\max _{u \in \pi_{(T, e)}} P(u)$.

Let $F$ be a spanning forest over $G$. For any $e=\{x, y\} \in(E \backslash E(F))$, we denote by $\pi_{(F, e)}$ the unique simple path in $F \backslash M$ such that:

- either $\pi_{(F, e)}=\langle x, y, \ldots, x\rangle$,

- or $\pi_{(F, e)}=\left\langle m_{1}, \ldots, x, y, \ldots, m_{2}\right\rangle$ with $m_{1}, m_{2} \in V(M)$.

Proposition A.2 [MinSF property]

Let $M$ be a subgraph of $G$.

The spanning forest $F$ over $G$ is a MinSF relative to $M$ over $G$ for $P$ if and only if $\forall e \in(E \backslash E(F)), P(e)=\max _{u \in \pi_{(F, e)}} P(u)$.

\section{Proof of proposition A.2:}

It was shown in [4] that a MinSF can be viewed as a minimum spanning tree in an enlarged graph where the markers and the connected components of $G$ are linked by additional edges with zero weight. As a consequence, properties on MinSFs (and in particular this theorem) are deduced from properties on minimum spanning trees.

Now, this property is preserved through the flooding maps according to the following proposition applied to the paths $\pi_{(F, e)}$ with $e \in(E \backslash E(F)$ ).

Proposition A.3 [Flooding maps property]

Let $\pi=\left\langle e_{0}, \ldots, e_{\ell-1}\right\rangle$ be a path in $E$ and let $e \in \pi$ such that $P(e)=$ $\max _{u \in \pi} P(u)$.

If $P^{\prime}$ is a flooding map of $P$ for $G$, then $P^{\prime}(e)=\max _{u \in \pi} P^{\prime}(u)$.

\section{Proof of proposition A.3:}

Let us prove the result by contradiction.

Assume that $P^{\prime}$ is a flooding map such that there exists an edge $e^{\prime} \in \pi$ with $P^{\prime}\left(e^{\prime}\right)>P^{\prime}(e)$. Then there exists $h \in \mathbb{R}^{+}$with $P^{\prime}\left(e^{\prime}\right)>h>P^{\prime}(e)$. Then the connected component $X$ of $G_{P^{\prime}}^{h}$ which contains $e$ does not contain $e^{\prime}$. Since $P^{\prime}$ 
is a flooding map, $X$ is a connected component of $G_{P}^{h}$. Since any edge $\tilde{e}$ of $\pi$ satisfies $P(\tilde{e}) \leq P(e) \leq P^{\prime}(e)<h$, all edges of $\pi$ are in $X$, and in particular $e^{\prime}$. This contradicts the premise.

As a consequence, if $F$ is a MinSF relative to $M$ over $G$ for $P$, then $F$ is a MinSF relative to $M$ over $G$ for $P^{\prime}$.

\section{B Proof of theorem 5.3}

Suppose that $C$ is a MinSF cut relative to $M$ for $P$ and let $P^{\prime}$ and $P^{\prime \prime}$ defined as in definition 4.3.

Since changing the weights of edges in $M$ does not affect the MinSF property, $C$ is a MinSF cut relative to $M$ for $P^{\prime}$. By theorem 5.2, we deduce that $C$ is also a MinSF relative to $M$ for $P^{\prime \prime}$ and, by theorem 5.1, a watershed relative to $M$ for $P$.

\section{Proof of theorem 6.1}

In the following of this proof, the extensions and forests considered are all relative to the subgraph $M$, and they are over graphs for which the set of vertices is $V=V(G)$, so that we introduce the following convenient notation.

\section{Notation:}

For a set of edges $E(M) \subseteq E^{\prime} \subseteq E=E(G)$ :

- an $E^{\prime}$-MaxSF is a MaxSF relative to $M$ over $\left(V, E^{\prime}\right)$.

- an $E^{\prime}$-spanning forest is a spanning forest relative to $M$ over $\left(V, E^{\prime}\right)$.

- an $E^{\prime}$-extension is an extension relative to $M$ over $\left(V, E^{\prime}\right)$.

- an $E^{\prime}$-spanning extension is a spanning extension relative to $M$ over $\left(V, E^{\prime}\right)$.

- an $E^{\prime}$-maximal extension is a maximal extension relative to $M$ over $\left(V, E^{\prime}\right)$.

Let $r=\min \left\{\frac{P\left(e_{i}\right)}{P\left(e_{j}\right)}\right.$ s.t. $\left(e_{i} ; e_{j}\right) \in E^{2}$ with $\left.P\left(e_{i}\right)>P\left(e_{j}\right)\right\}$ and take

$$
\beta=\frac{\log |E|}{\log r}
$$

Let $C$ be a min-cut relative to $M$ for $P^{[\alpha]}$ with $\alpha \geq \beta$. Let $F$ be a $E$-MaxSF. Let $\tilde{F}$ be a $\bar{C}$-MaxSF, where we recall $\bar{C}=E \backslash C$. Let $P(X)$ be the weight of a subgraph $X$ of $G$. 
To prove the result, i.e. that $\tilde{F}$ is a $E$-MaxSF, we will show that:

(i) $P(\tilde{F})=P(F)$,

(ii) $\tilde{F}$ is a $E$-spanning forest.

To obtain (i), we will prove that $F$ and $\tilde{F}$ have the same number of edges of each type of weights.

Let $\mathcal{P}=\left\langle p_{1}, \ldots, p_{\ell}\right\rangle$ be the different weights of the edges in $G$, such that:

$$
\begin{aligned}
& \forall i \in\{1, \ldots, \ell\}, \exists e \in E \mid P(e)=p_{i} \\
& \forall e \in E, \exists i \in\{1, \ldots, \ell\} \mid P(e)=p_{i}
\end{aligned}
$$

and

$$
\forall i, j \in\{1, \ldots, \ell\}, \text { if } i \neq j \text { then } p_{i} \neq p_{j},
$$

presented in decreasing order with

$$
p_{1}>\cdots>p_{\ell}
$$

We say that $\mathcal{P}$ is the induced sequence of $G$.

Let $n_{h}(X)$ be the number of edges of weight $p_{h}$ of a subgraph $X$ of $G$. Then, we obviously have

$$
|E(X)|=\sum_{h=1}^{\ell} n_{h}(X)
$$

and the weight of $X$ may be expressed as

$$
P(X)=\sum_{h=1}^{\ell} n_{h}(X) p_{h}
$$

To get (i), it suffices to show that for any $h \in\{1, \ldots, \ell\}$,

$$
n_{h}(F)=n_{h}(\tilde{F}) .
$$

We define

$$
E^{h}=\left\{e \in E ; P(e) \geq p_{h}\right\} \cup E(M)
$$

and introduce

$$
\begin{aligned}
& F^{h}=\left(V, E(F) \cap E^{h}\right) \\
& \tilde{F}^{h}=\left(V, E(\tilde{F}) \cap E^{h}\right)
\end{aligned}
$$

Figure C.1 illustrates these notations. 


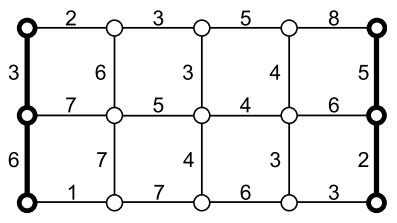

(a)

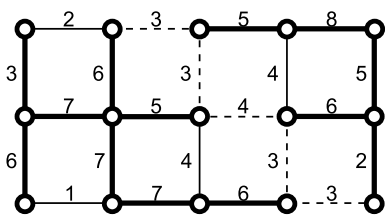

(b)

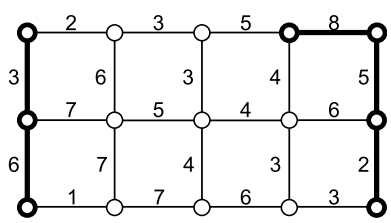

(c)

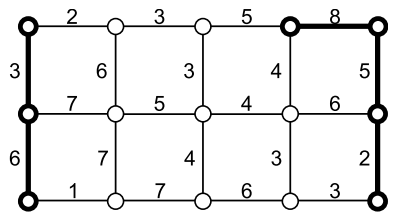

(f)

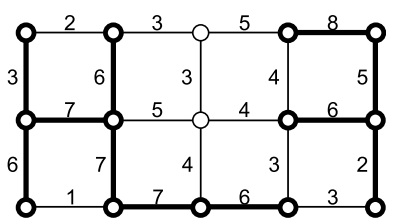

(d)

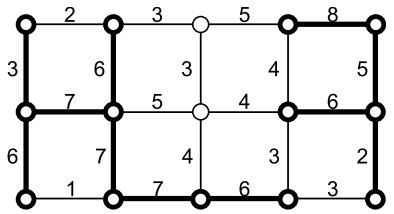

(g)

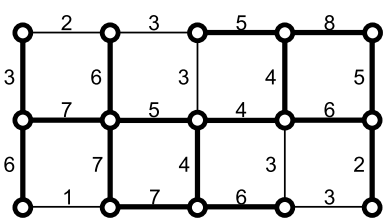

(e)

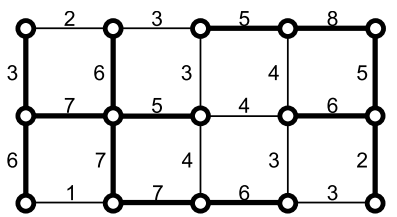

(h)

Figure C.1. Graph $G$ and map $P$ (which induced sequence is $\langle 9,8,7,6,5,4,3,2,1\rangle$ ) with in bold: (a) a subgraph $M$; (b) a MaxSF relative to $M$ for $P$; (c) $G^{2}\left(p_{2}=8\right)$; (d) $G^{4}\left(p_{4}=6\right) ;\left(\right.$ e) $G^{6}\left(p_{6}=4\right) ;\left(\right.$ f) $F^{2} ;\left(\right.$ g) $F^{4} ;(\mathrm{h}) F^{6}$.

Note that $F=F^{\ell}$ and $\tilde{F}=\tilde{F}^{\ell}$. To obtain equation (C.8), we will prove by induction on $k$ that for any $1 \leq h \leq k$,

$$
n_{h}\left(F^{k}\right)=n_{h}\left(\tilde{F}^{k}\right) .
$$

The proof of equation (C.12) requires the three following lemmas.

\section{Lemma C.1}

For any $h \in\{1, \ldots, \ell\}, F^{h}$ is a $E^{h}$-spanning forest.

\section{Proof of lemma C.1:}

Let $G^{h}=\left(V, E^{h}\right)$. We start with two obvious statements:

- Any connected component of $M$ is included in a connected component $K_{F^{h}}$ of $F^{h}$, since by definition of $F^{h}$ we have $E(M) \subseteq F^{h}$.

- The connected graph $K_{F^{h}}$ cannot contain more than one connected component of $M$ since, otherwise, one of the connected components of $F$ would contain more than one connected component of $M$, which is impossible.

So to prove that $F^{h}$ is a $E^{h}$-extension, it suffices to check that for any connected component $K_{G^{h}}$ of $G^{h}$ (whether $V\left(K_{G^{h}}\right) \cap V(M)$ is empty or nonempty), the graph $K_{G^{h}} \cap F^{h}$ is connected. We will prove it by contradiction. Assume that it is disconnected. Then there exists $e=\{x, y\} \in E\left(K_{G^{h}}\right)$ such 
that $e \notin E\left(F^{h}\right)$. Since $P(e) \geq p_{h}$, then we also have $e \notin E(F)$. So there is either a simple path in $F$ between $x$ and $y$ containing an edge $e^{\prime} \in E \backslash E^{h}$ or a simple path in $F$ from $x$ to a node of $M$ containing an edge $e^{\prime} \in E \backslash E^{h}$. In both cases, if we remove $e^{\prime}$ and add $e$ to $F$, we obtain a $E$-spanning forest whose weight is greater than the one of $F$ (since $P(e) \geq p_{h}>P\left(e^{\prime}\right)$ ). This contradicts the fact that $F$ is a $E$-spanning forest with maximal weight. So $F^{h}$ is a $E^{h}$-extension.

Now let $e^{\prime \prime} \in E\left(F^{h}\right)$. The subgraph $\left(V, E\left(F^{h}\right) \backslash\left\{e^{\prime \prime}\right\}\right)$ is not a $E^{h}$-extension since one can check that otherwise $\left(V, E(F) \backslash\left\{e^{\prime \prime}\right\}\right)$ would be a $E$-extension, which contradicts the fact that $F$ is a $E$-spanning forest. So $F^{h}$ is a $E^{h}$ spanning forest.

\section{Remark C.1}

Using the same kind of construction as the one used to prove that $F^{h}$ is a $E^{h}$-extension, one can prove that $F^{h}$ is in fact a $E^{h}$-MaxSF but we will not use it here.

\section{Lemma C.2}

For any $h \in\{1, \ldots, \ell\}, \tilde{F}^{h}$ is a $E^{h}$-spanning forest.

\section{Proof of lemma C.2:}

Let $\tilde{E}^{h}=E^{h} \backslash C, \tilde{G}^{h}=\left(V, \tilde{E}^{h}\right)$ and $G^{h}=\left(V, E^{h}\right)$. Let us prove that $\tilde{G}^{h}$ is a $E^{h}$-extension. We start with noting that:

- any connected component of $M$ is included in a connected component $K_{\tilde{G}^{h}}$ of $\tilde{G}^{h}$, since the cut $C$ relative to $M$ over $G$ does not cut edges of $E(M)$,

- the connected graph $K_{\tilde{G}^{h}}$ cannot contain more than one connected component of $M$ since, otherwise, one of the connected components of $\tilde{G}^{\ell}=$ $(V, E \backslash C)$ would contain more than one connected component of $M$, which is impossible by definition of $C$.

So to prove that $\tilde{G}^{h}$ is a $E^{h}$-extension, it suffices to check that for any connected component $K_{G^{h}}$ of $G^{h}$ (whether $V\left(K_{G^{h}}\right) \cap V(M)$ is empty or nonempty), the graph $K_{G^{h}} \cap \tilde{G}^{h}$ is connected. We will prove it by contradiction. Assume that it is disconnected. Then there exists $e=\{x, y\} \in E\left(K_{G^{h}}\right)$ such that $e \in C$, i.e. $e$ is in the min-cut $C$ relative to $M$ over $G$. Since the vertices $x$ and $y$ are not both in $V(M)$, starting with $C$, we can build a cut $C^{\prime}$ relative to $M$ over $G$ by removing the edge $e$ and by adding edges from $E \backslash E^{h}$ in order to ensure that $C^{\prime}$ is a cut. For $P^{[\alpha]}$, the total weight of the edges added to $C \backslash\{e\}$ is smaller than $P^{[\alpha]}(e)$, since from equation (C.1) and $\alpha \geq \beta$, we 
have

$$
\begin{aligned}
\alpha & \geq \frac{\log |E|}{\log \frac{p_{h}}{p_{h+1}}}, \\
\log \frac{p_{h}^{\alpha}}{p_{h+1}^{\alpha}} & \geq \log |E|, \\
p_{h}^{\alpha} & \geq|E| p_{h+1}^{\alpha}
\end{aligned}
$$

and, consequently,

$$
P^{[\alpha]}(e)=[P(e)]^{\alpha} \geq p_{h}^{\alpha} \geq|E| p_{h+1}^{\alpha}>\sum_{i=h+1}^{\ell} n_{i}(G) p_{i}^{\alpha} .
$$

So the cut $C^{\prime}$ would satisfy $P\left(C^{\prime}\right)<P(C)$, which is impossible. Therefore $\tilde{G}^{h}$ is a $E^{h}$-extension.

Let us now prove by contradiction that it is also a $E^{h}$-maximal extension. Assume that there exists an edge $e$ which you could add to $\tilde{G}^{h}$ such that $\check{G}^{h}=\left(V, E\left(\tilde{G}^{h}\right) \cup\{e\}\right)$ is also a $E^{h}$-extension. Let $\check{C}^{h}=E\left(G^{h}\right) \backslash E\left(\check{G}^{h}\right)$. Then, starting with $\check{C}^{h}$, you can build a cut $C^{\prime}$ relative to $M$ over $G$ by adding edges from $E \backslash E^{h}$ in order to ensure that $C^{\prime}$ is a cut. Using the same argument than in equation (C.14), one can check that this would lead to $P\left(C^{\prime}\right)<P(C)$, which is impossible. So $\tilde{G}^{h}$ is simultaneously a maximal extension relative to $M$ over $G^{h}$ and a maximal extension relative to $M$ over $\tilde{G}^{h}$.

Since a maximal extension is a spanning extension, we deduce from the second way to define a spanning forest (in definition 1.3 ) that $\tilde{F}^{h}$ is a $E^{h}$-spanning forest.

\section{Remark C.2}

As a by-product of this proof, we have that $E^{h} \cap C$ is a min-cut relative to $M$ $\operatorname{over}\left(V, E^{h}\right)$.

\section{Lemma C.3}

Let $E(M) \subseteq E^{\prime} \subseteq E$. Any $E^{\prime}$-spanning forest has the same number of edges.

\section{Proof of lemma C.3:}

It suffices to check that the number of edges of a $E^{\prime}$-spanning forest $F^{\prime}$ is exactly

$$
\begin{aligned}
& \left|E\left(F^{\prime}\right)\right|=|E(M)| \\
& +\sum_{H}\{|V(H)|-N(H \cap M)-\max (|V(H \cap M)|-1,0)\},
\end{aligned}
$$

where the summation is taken over the connected components of $\left(V, E^{\prime}\right)$, and $N(X)$ denotes the number of connected components of $X$. 
Now that we have proved our three lemmas, let us go back to the proof of equation (C.12) as follows.

Proof by induction on $k$ of equation (C.12):

- For $k=1$ : from lemmas C.1, C.2 and C.3, $F^{1}$ and $\tilde{F}^{1}$ have the same number of edges. Now since in both subgraphs the only edges of weight smaller than $p_{1}$ are exactly the edges in $M$ of weight smaller than $p_{1}$, we obtain $n_{1}\left(F^{1}\right)=n_{1}\left(\tilde{F}^{1}\right)$.

- Assume now that equation (C.12) holds for $k$. Let us show that it then holds for $k+1$. For any $1 \leq h \leq k$, we have

$$
n_{h}\left(F^{k+1}\right)=n_{h}\left(F^{k}\right)=n_{h}\left(\tilde{F}^{k}\right)=n_{h}\left(\tilde{F}^{k+1}\right),
$$

where the second equality uses equation (C.12) for $k$. Now, from lemmas C.1, C.2 and C.3, $F^{k+1}$ and $\tilde{F}^{k+1}$ have the same number of edges. Since in both subgraphs the only edges of weight smaller than $p_{k+1}$ are exactly the edges in $M$ of weight smaller than $p_{k+1}$, we obtain $n_{k+1}\left(F^{k+1}\right)=n_{k+1}\left(\tilde{F}^{k+1}\right)$, which finishes the induction.

So the proof of (i) is finished. For (ii), it suffices to note that, since we have $E=E^{\ell}$ and $F=F^{\ell}$, it is a direct consequence of lemma C.2.

\section{Remark C.3}

In the proof of theorem 6.1, the fact that $\alpha$ is large enough is only required to ensure that for any $h \in\{1, \ldots, \ell-1\}, G^{h}=\left(V, E^{h} \backslash C\right)$ is a $E^{h}$-extension. In image processing, it often occurs that the min-cut relative to $P^{[\gamma]}$ with small $\gamma$ (typically $\gamma=1$ ) oversmooths the contours, and that we want to try higher values for $\gamma$ to obtain tighter contours.

The previous remark can be used to speed up computations of the min-cut for $P^{\left[\gamma^{\prime}\right]}$ with $\gamma^{\prime}>\gamma$ when the min-cut for $P^{[\gamma]}$ has already been computed. 


\section{References}

[1] Y. Boykov, O. Veksler, R. Zabih, Fast approximate energy minimization via graph cuts, IEEE Transactions on Pattern Analysis and Machine Intelligence 23 (11) (2001) 1222-1239.

[2] S. Beucher, C. Lantuéjoul, Use of watersheds in contour detection, in: Proceedings of the International Workshop on Image Processing Real-Time Edge and Motion Detection/Estimation, 1979.

[3] J. Cousty, G. Bertrand, L. Najman, M. Couprie, Watershed cuts, in: Proceedings of the 8th International Symposium on Mathematical Morphology, 2007, pp. 301-312.

[4] J. Cousty, G. Bertrand, L. Najman, M. Couprie, Watershed cuts: minimum spanning forests and the drop of water principle, IEEE Transactions on Pattern Analysis and Machine Intelligence 31 (8) (2009) 1362-1374.

[5] F. Meyer, Minimum spanning forests for morphological segmentation, in: Proceedings of the 2nd International Conference on Mathematical Morphology and its Applications to Image Processing, 1994, pp. 77-84.

[6] C. Allène, J.-Y. Audibert, M. Couprie, J. Cousty, R. Keriven, Some links between min-cuts, optimal spanning forests and watersheds, in: Proceedings of the 8th International Symposium on Mathematical Morphology, INPE, 2007, pp. 253-264.

[7] G. Bertrand, On topological watersheds, Journal of Mathematical Imaging and Vision 22 (2-3) (2005) 217-230.

[8] T. H. Cormen, C. E. Leiserson, R. L. Rivest, Introduction to algorithms, second edition, MIT Press, 2001.

[9] B. Korte, J. Vygen, Combinatorial optimization: theory and algorithms, Algorithms and Combinatorics, Springer, 2000.

[10] F. Meyer, S. Beucher, Morphological segmentation, Journal of Visual Communication and Image Representation 1 (1) (1990) 21-46.

[11] L. Vincent, P. Soille, Watersheds in digital spaces: an efficient algorithm based on immersion simulations, IEEE Transactions on Pattern Analysis and Machine Intelligence 13 (6) (1991) 583-598.

[12] F. Meyer, L. Najman, Morphologie mathématique 1 : approches déterministes, Traité IC2, série signal et image, Hermès Science Publications, 2008, Ch. Segmentation, arbre de poids minimum et hiérarchies, pp. 201-232.

[13] J. Serra, C. Vachier, F. Meyer, Morphologie mathématique 1 : approches déterministes, Traité IC2, série signal et image, Hermès Science Publications, 2008, Ch. Nivellements, pp. 173-200. 
[14] L. Vincent, Morphological grayscale reconstruction in image analysis: applications and efficient algorithms, IEEE Transactions on Image Processing 2 (2) (1993) 176-201.

[15] B. Chazelle, A minimum spanning tree algorithm with inverse-Ackermann type complexity, Journal of the Association for Computing Machinery 47 (2000) $1028-1047$.

[16] L. Najman, M. Schmitt, Geodesic saliency of watershed contours and hierarchical segmentation, IEEE Transactions on Pattern Analysis and Machine Intelligence 18 (12) (1996) 1163-1173.

[17] R. Diestel, Graph theory, Graduate Texts in Mathematics, Springer, 1997.

[18] L. R. S. Ford, D. R. Fulkerson, Maximal flow through a network, Canadian Journal of Mathematics 8 (1956) 399-404.

[19] Y. Boykov, O. Veksler, R. Zabih, A new algorithm for energy minimization with discontinuities, in: Proceedings of the 2nd International Workshop on Energy Minimization Methods in Computer Vision and Pattern Recognition, SpringerVerlag, London, UK, 1999, pp. 205-220.

[20] E. Dahlhaus, D. S. Johnson, C. H. Papadimitriou, P. D. Seymour, M. Yannakakis, The complexity of multiway cuts, in: Proceedings of the 24th Annual Association for Computing Machinery Symposium on the Theory of Computing, Association for Computing Machinery, New York, NY, USA, 1992, pp. 241-251. 